\title{
A Parametric Model for Analysing Atherosclerotic Arteries: on the FSI coupling
}

\author{
M. Cilla ${ }^{a, b}$, I. Borrás $^{b}$, E. Peña ${ }^{b, c}$, M.A. Martínez ${ }^{b, c}$, M. Malvè ${ }^{b, c, d}$ \\ ${ }^{a}$ Julius Wolff Institute for Biomechanics and Musculoskeletal Regeneration - \\ Universitaetsmedizin Berlin \\ ${ }^{b}$ Aragón Institute of Engineering Research (I3A). University of Zaragoza. \\ C/María de Luna s/n, E-50018 Zaragoza, Spain \\ ${ }^{c}$ Centro de Investigación Biomédica en Red en Bioingeniería Biomateriales y \\ Nanomedicina (CIBER-BBN) C/Poeta Mariano Esquillor s/n, E-50018 Zaragoza \\ ${ }^{d}$ Public University of Navarra. Department of Mechanical Engineering, Energetics \\ and Materials, Campus Arrosadía, E-31006 Pamplona, Spain \\ E-mail: mauro.malve@unavarra.es
}

\begin{abstract}
There are many evidences that coronary plaque is not only dependent on the formation and progression of atherosclerosis, but also on the vascular remodelling response. If the local wall shear stress is low, a proliferative plaque may develop. Local inflammatory response will stimulate the formation of a plaque prone to rupture with superimposed thrombus formation (vulnerable plaque). Furthermore, the role of the wall shear stress in the genesis and the development of atherosclerotic diseases has been recently intensively investigated, examining its relationship with the presence of lesions and the intima media thickness. Due to the important role of pulsating blood flow, pressure and hemodynamics factors in atheroma growth, a Fluid Structure Interaction (FSI) parametric study of a 3D atherosclerotic artery has been carried out, with aim of studying the main geometrical risk factors in terms of plaque vulnerability.
\end{abstract}


Key words: Atherosclerosis, vulnerable plaque, Lipid core, coronary disease, fluid-solid interaction, finite element method

\section{Introduction}

Cardiovascular diseases related to atherosclerosis are nowadays recognized as one of the principal causes of death worldwide. Atherosclerosis is a pathological process in which plaques, consisting of deposits of cholesterol and other lipids, calcium and large inflammatory cells called macrophages, connective tissue and other substances, are built up in the inner lining of the arterial walls of the arteries [1]. This fatty tissue, known as atheroma, cause narrowing or stenosis of the lumen, rupture or erosion of the arterial wall, hardening of the arteries and loss of their elasticity, which leads to an eventual or complete reduction in the blood flow through the vessels. Nevertheless, the most serious damage occurs when the plaque becomes fragile and ruptures (vulnerable plaque). Plaque rupture causes the formation of blood clots that can block blood flow or break off and travel to another part of the circulatory system producing heart attacks and strokes among other pathologies. Plaque disruption tends to occur at points where the plaque surface is the weakest and most vulnerable. These locations coincide with points where stresses, which are affected mainly by tissue properties and plaque geometry, are concentrated [2]. Traditionally, coronary atheroma plaques with a thin fibrous cap thickness and high stenosis ratio have been considered as prone to rupture. However, several pathological and biomechanical studies [3-6] have provided morphological descriptions of the vulnerable risk, and it can be concluded that the fibrous cap thickness alone is not a sufficiently accurate predictor for plaque stability. The lipid core length and width are also very important parameters on vulnerability $[4,6,7]$. Nevertheless, apart from plaque morphology, there are other factors which could play a key role in the initiation of failure such as the presence of microcalcifications [8-11] or hemodynamic features such as blood flow separation and circulation. Within this context, the com- 
plex blood flow dynamics in the arterial system has been also recognized to have a key role in the initiation and development of plaques $[12,13]$. In particular, low endothelial shear stress of the plaque is recognized to be prone to develop plaque while the high shear stress region is indicated as responsible of destabilization of the cap covering the plaque [13]. Weakening of the cap will eventually result in plaque rupture. Computational Fluid Dynamics (CFD) has been applied in diseased and non-diseased arteries. Until now, a variety of factors related to hemodynamics in plaque region have been proposed to quantify flow disturbances as potential predictors for arterial wall dysfunction, including the Time Averaged Wall Shear Stress (TAWSS) [14-16], the Oscillatory Shear Index (OSI), the Relative Residence Time (RRT), the WSS Spatial Gradient (WSSG) or the WSS Angle Gradient (WSSAG) [17]. Within the atherosclerosis on coronary arteries, studies on these diseased arteries are normally focused on hemodynamic aspects, neglecting the interaction between blood flow and arterial walls, evaluating the WSS at the rigid walls and connecting it with atheromatous pathologies [18,14,19-21]. While several FSI simulations has been performed in 2D models [22,23],3D models have also been utilized to predict wall shear stress and wall stress patterns in healthy subjects [24]. Tang and coworkers [25-31] extensively work on FSI approach of atheromatous plaque using patient specific-based models and performing statistical analysis for carotid and coronary arteries. Li et al. [32] constructed an idealized 3D atheroma plaque model with varying stenosis degree of $30 \%, 50 \%$ and $70 \%$, to study the wall motion in plaque throat. The results suggested that severe stenosis may inhibit wall motion. Ohayon et al. [33] quantified the WSS and the stiffness in coronary bifurcations and investigated the correlations with atheroma plaque sites.

In this study, we carried out a 3D FSI parametric study of an idealized coronary vessel with aim to quantify and investigate the biomechanical interaction between the most influential geometrical factors of the vessel in the plaque rupture: (i) the fibrous cap thickness; (ii) the stenosis ratio; (iii) the lipid core width and (iv) the lipid core length. Both distributions on the solid part model such as the maximum principal stress (MPS) or the blood vessel deformations, and on the fluid part such 
as the wall shear stress (WSS), time average wall shear stress (TAWSS), residual resident time (RRT), oscillatory shear index (OSI) and average wall shear stress gradient (AWSSG) have been obtained. Furthermore, the influence of including the fluid effects has been shown by comparing the results of a 3D parametric study published by the same authors [6] with the obtained results on this FSI analysis.

\section{Materials and Methods}

\subsection{Model Geometry}

An idealized model of artery with an eccentric stenosis was used to carry out an FSI 3D parametric study in order to investigate the influence of the essential geometrical factors related to the plaque rupture such as lipid core locations and plaque morphology. Atherosclerotic vessel morphology and its dimensions were obtained from Versluis et al. [34] and Bluestein et al. [8]. The selected dimensions represent a standard coronary artery. In particular, for the considered model, the vessel external diameter was $4 \mathrm{~mm}$, the wall thickness was $0.5 \mathrm{~mm}$ and the vessel length was $20 \mathrm{~mm}$. The plaque consists of a lipid pool, which is enveloped with a thin fibrous cap and located in the inner surface of the vessel. The healthy vessel wall is composed of two layers; media and adventitia, however the media was considered as fibrotic layer in those areas with atheroma plaque. Therefore, the solid model was divided in 4 parts: adventitia, media, plaque and lipid core, and the fluid part corresponds to the volume inside of the solid model part (Fig. 1). The parametric study evaluates a series of idealized plaque morphology models representing different stages and variations of the atherosclerotic lesion growth. The most influential geometric parameters were extracted from previous works $[3,5,6]$. These are: the fibrous cap thickness, $(f c)$, the stenosis ratio in the central cross-section $(s r)$ - which is obtained by dividing the lumen radius by the lumen radius of a normal artery $(R=1.5 \mathrm{~mm}), \operatorname{sr}(\%)=\frac{r(\mathrm{~mm})}{R(\mathrm{~mm})} 100-$, the lipid core length $(l)$ and the lipid core 
Table 1

Geometrical parameter values used to generate the 17 parametric 3D models (the values for the reference case are written in bold

\begin{tabular}{lccccc} 
Parameters & 1 & 2 & $\mathbf{C b}$ & 4 & 5 \\
\hline fc $(m m)$ & 0.025 & 0.035 & $\mathbf{0 . 0 5}$ & 0.1 & 0.15 \\
sr $(m m)$ & 46.7 & 53.3 & $\mathbf{5 6 . 7}$ & 60 & 66.7 \\
l $(m m)$ & 1 & 2 & $\mathbf{4}$ & 6 & 8 \\
w $(\%)$ & 30 & 45 & $\mathbf{6 0}$ & 75 & 90 \\
\hline
\end{tabular}

width $(w)$. The lipid core width $(w)$ was defined as the ratio between the percentage of the atheroma plaque width $\left(w_{1}\right)$ and the distance from the inner point of the lipid core to the outer point of the fibrotic plaque $\left(w_{2}\right), a(\%)=\frac{w_{1}(\mathrm{~mm})}{w_{2}(\mathrm{~mm})} 100$ (see Fig. 1). Five realistic morphological data were considered of each parameter: the fibrous cap thickness $(0.025 \leq e \leq 0.15$, in $\mathrm{mm})$, the lumen radio $(0.7 \leq r \leq 1$, in $\mathrm{mm})$, the lipid core length $(1 \leq l \leq 8$, in $\mathrm{mm})$ and the lipid core width $(30 \leq a \leq 90$, in $\%)$. Combining these five values for each parameter, a total of 17 idealized models of artery with atherosclerotic plaque were obtained. It has to be noted that, considering the intermediate value of each geometrical parameter, the reference model or base case $(\mathbf{C b})$ is defined. The remaining 16 models were obtained changing only one parameter each time, from the range described above keeping unchanged the another three parameters (see Tab. 1). Grid size for solid and fluid part are approximately 125000 and 800000 tetrahedral elements, with 25000 and 140000 nodes respectively. Figure 2 shows both the solid and the fluid mesh and geometry artery model. Figures 2.a and 2.b, respectively, correspond to one of the 17 computed models. 


\section{Table 2}

Material parameters of the SEF developed by Holzapfel et al. [35] used for the adventitia $(\mathrm{A})$, the media $(\mathrm{M})$, the atheroma plaque $(\mathrm{P})$ and the lipid core $(\mathrm{L})$

\begin{tabular}{ccccccc} 
& $\mu(k P a)$ & $k_{1}(k P a)$ & $k_{2}(-)$ & $\rho(-)$ & $\beta\left(^{\circ}\right)$ & $\varepsilon(-)$ \\
\hline $\mathrm{A}$ & 1.99 & 89.97 & 97.30 & 0.77 & \pm 36 & 0.036 \\
$\mathrm{M}$ & 1.02 & 25.11 & 8.58 & 0.32 & \pm 30.28 & 0.019 \\
$\mathrm{P}$ & 10.99 & 950.88 & 5.00 & 0 & - & 0.054 \\
$\mathrm{~L}$ & 0.01 & 66.24 & 4.99 & 0 & - & 0.031 \\
\hline
\end{tabular}

\subsection{Material properties}

\subsubsection{Arterial wall}

The material properties of the solid part were based on the experimental data of Holzapfel et al. [35] for the media and adventitia layers and Versluis et al. [34] for the lipid core and the fibrotic plaque. The material constants were fitted by the strain energy function (SEF) proposed by Holzapfel et al. [35] (see Tab. 2):

$$
\Psi=\mu\left[I_{1}-3\right]+\frac{k_{1}}{2 k_{2}} \sum_{i=4,6}\left[\exp \left(k_{2}\left[[1-\rho]\left[I_{1}-3\right]^{2}+\rho\left[I_{i}-1\right]^{2}\right]\right)-1\right]
$$

where $\mu>0$ and $k_{1}>0$ are stress-like parameters and $k_{2}>0$ and $0 \leq \rho \leq 1$ are dimensionless parameters (when $\rho=1$ the fibres are perfectly aligned and when $\rho=0$ the fibres are randomly distributed and the material is considered as isotropic), $I_{1}$ is the first invariant, and $I_{4}$ and $I_{6}$ are invariants which depend on the direction of the family of fibres at a material point [35]. Healthy wall was modelled as an anisotropic material with two families of fibres, oriented at $\pm 36^{\circ}$ and $\pm 30.28^{\circ}$, with respect to the circumferential direction, for the adventitia and media layer, respectively. 


\subsection{Boundary conditions}

The blood flow was modeled as laminar, incompressible (density, $\rho_{f}=1050 \frac{\mathrm{Kg}}{\mathrm{m}^{3}}$ ) and non-Newtonian, using the Carreau constitutive law. The Carreau model assumes that the viscosity of blood, $\mu$, varies according to the following equation:

$$
\mu=\mu_{\infty}+\left(\mu_{0}-\mu_{\infty}\right) \cdot\left(1+A_{c} \dot{\gamma}_{i j}^{2}\right)^{m_{c}}
$$

where $\mu_{0}$ and $\mu_{\infty}$ are low and high shear rate asymptotic values, while the parameters $A_{c}$ and $m$ control the transition region size [36]. The Carreau blood model predicts decreasing viscosity at high strain. For this study we used the experimental values provided in [36] and used also by other authors [37,38]: $\mu_{0}=0.056 \frac{\mathrm{N} \cdot \mathrm{s}}{\mathrm{m}^{2}}$, $\mu_{\infty}=0.00345 \frac{N \cdot s}{m^{2}} A_{c}=10.975$ and $m_{c}=-0.3216$. As usual for FSI analyses, mixed velocity-pressure boundary conditions are required in order to correctly compute flow features and structural stresses, strains and displacements. Therefore, for this study, physiological coronary flow and pressure waveforms are necessary. These conditions, imposed at the inlet and at the outlet of the model respectively were extracted from the experimental data of Davies et al. [39]. Furthermore, for the solid model, in order to avoid the rigid body movement of the arterial model, the displacements were constrained at the ends of the blood vessel [16]. Finally, in order to dump the influence of the boundary conditions imposition, five diameter inlet and outlet extensions were added to the models.

\section{Results}

\subsection{Stresses distribution in the arterial wall}

The MPS distribution occurring on each of the considered 17 models were studied. Figure 3 shows the obtained 17 MPS contour maps in the cross central section of each model at the instant of time corrsponding to the peak pressure $(t=0.36 \mathrm{~s})$. These contour maps show that the maximum MPS is produced in the fibrous cap region in all models. Furthermore, it should be noted that any of the 17 studied models 
exceeds the threshold stress of $247 \mathrm{kPa}$ which has been suggested in the literature to define atheroma plaque vulnerability $[6,40]$. For the sake of clarity, Fig. 4 shows the maximum MPS at peak flow during diastole $(t=0.36 \mathrm{~s})$ in order to compare the variation of this stress versus each of the geometrical parameters considered in this 3D FSI parametric study. Thereby, the tendency of the maximum MPS versus each geometrical parameter can be observed. Figure 4.a shows the MPS evolution with fibrous cap variation, showing a dramatically increase of MPS as this parameter decreases. Comparing the models with $0.15,0.1$ and $0.05 \mathrm{~mm}$ of cap thickness, the maximum MPS values obtained are 64.3, 73.98 and $139.11 k P a$, respectively. For the stenosis ratio and lipid core parameters, Fig. 4.b and 4.d, respectively, the maximum MPS increases slightly as these parameters increase. Finally, for the lipid core length, Fig.4.c, the maximum MPS increases as the lipid core length increases until $4 \mathrm{~mm}$, and after that, the maximum MPS remains practically constant. Furthermore, as aforementioned, any conclusion about vulnerability limits could be obtained from these results since any model exceed the vulnerability threshold stress value of $247 \mathrm{kPa}[6,40]$. Figures 3 and 4 show the MPS contour maps and the maximum MPS, respectively, of each studied model at $t=0.36 \mathrm{~s}$, which corresponds to the maximum pressure instant. However, since the applied pressure varies with the time, the maximum MPS also varies along the imposed physiological cardiac cycle. Therefore, Fig. 5 illustrates the tendency of the maximum MPS along this cycle $(\Delta t=0.9 \mathrm{~s})$. The trend observed in the maximum MPS variation along the time is similar to the applied time varying pressure cycle, being the maximum MPS reached at $0.36 s$ which corresponds to the moment of maximum pressure applied.

\subsection{Arterial deformation}

A previous study from the same authors [6] provided some guidelines to assess the atheroma plaque vulnerability according to four of the most influential geometrical parameters on the risk of plaque rupture by means of FE structural analysis, and assuming constant internal pressure. The geometry and general dimensions, the boundary conditions and the material parameters used in that study where the same 
than those defined in this study. Thus, in order to assess the influence of including the blood flow effects in the model, the results of both studies have been compared. It should be noted that the maximum pressure reached in the application of the physiological coronary cycle for the FSI study is equivalent to that used as internal constant pressure (140 $\mathrm{mmHg}$ or $18.3 \mathrm{kPa}$ ) for the $\mathrm{FE}$ study so that the comparison has been performed at this at peak diastole $(t=0.36 \mathrm{~s})$. The results, looking at the MPS contour maps in the cross central section of the reference case model, showed similar distributions. In addition, maximum MPS value was analogous and located at the same location of the fibrous cap for both models. This clearly demonstrates that the fluid-structure interaction approach provides for this study very similar results in term of MPS, even taking into account the effect of the fluid, neglected in $[6]$.

Plaque rupture may occur when the plaque structure cannot resist the hemodynamic blood pressure and shear stress $[3,5]$. Hemodynamic blood flow acts in fact as an extrinsic factor exerting pressure and shear stress on the plaque. The deformation of the plaque in turn will affect blood flow. Figure 6 shows the undeformed and maximum deformed model geometries for one of the 17 studied models (base case) in order to observe the differences between both states. Furthermore, the contour map of the maximal principal strain, which corresponds to the circumferential direction, (in logarithmic scale) for the instant of maximum pressure applied $(t=0.36 \mathrm{~s})$ are also shown. Observing this contour map, it is visible how the maximum maximal principal strain occurs in the artery wall close to the ends of the artery. As expected, this fact is related to the great stiffness of the central zone of the model due to the presence of the atheroma plaque, allowing more radial expansion in zones without atherosclerotic lesions. However, in order to predict atheroma plaque rupture, the maximal principal strain should be observed in the critical zones of the model, i.e, in the internal surface of the atheroma plaque. Figure 7 depicts the maximum percentage deformation of the 17 studied models. Furthermore, the maximum deformation of the atheroma plaque is around $15 \%$ and it is quite similar for all the considered models, ranging from 11.6 to $16 \%$, being these deformations allowable for an artery 
$[41,5]$. The high maximum principal strain presented in these models and the difference between original and deformed configurations (Fig. 6) supports the use of FSI analysis to get more accurate measures of structural and hemodynamic results. A trend is observed between the parameters and the maximum principal deformation since it increases as the lipid core width and length and the stenosis ratio increase, but on the contrary, it decreases as the fibrous cap thickness increases. Additionally, since the deformations are directly related to the stresses, this observed trend is analogous than that found for the case of the maximum MPS. Furthermore, the maximum deformation of the atheroma plaque is quite similar for all the considered models, varying around a $6 \%$ for the extreme cases, being these deformations allowable as an artery deformation. Notwithstanding, the maximum maximal principal strain varies from one to another parameter, reaching deformation variations of $4.5 \%$ and $5 \%$, for the fibrous cap thickness and for the lipid core length respectively, which are slightly higher than for the other two studied parameters (3\%).

\subsection{Hemodynamic Factors}

Due to the importance of diverse hemodynamic factors in the atherosclerosis development, the study of several hemodynamic variables such as wall shear stress (WSS), time average wall shear stress (TAWSS), oscillatory shear index (OSI), relative resident time (RRT) and average wall shear stress gradient (AWSSG) has been carried out. These quantities, well known in the literature of the field, are computed in this study according to [42]. Since the WSS distribution in the studied artery model mostly depends on the stenosis severity, similar WSS contour maps have been obtained for models with the same value of stenosis ratio (see Fig. 6), although the other three parameters, which modify the geometry of the atheroma plaque but not the internal geometry of the blood vessel, slightly varies from one to another model. Therefore, only 5 WSS patterns have been found in this study, corresponding to the reference case and the four variations of the stenosis ratio respect to the base case. Concerning the WSS as expected we found a region characterized by a high WSS value around the stenosis zone (atheroma plaque area) caused by the blood flow impacting against the arterial wall, founding greater WSS values as the stenosis 
ratio decreases. This WSS difference rises from $45.8 \mathrm{~Pa}$ to $28.9 \mathrm{~Pa}$ (Fig. 8), for the most and less stenosis ratio considered $(s r=46.7 \%$ and $s r=66.7 \%)$, respectively. Although a direct link between the plaque rupture and high shear stress has not been established, as suggested in literature, this high WSS could induce thinning of the fibrous cap and may destabilize the plaque [43]. However, the minimum WSS occurs in the pre and post stenotic areas, corresponding to the zones where blood recirculation areas are found. The minimum WSS remains practically constant despite of the stenosis ratio variation, since the minimum WSS varies from $0.78 \mathrm{~Pa}$ to $0.1 \mathrm{~Pa}$, for the most and less severe atherosclerotic lesion considered, respectively. This small variations are related to the inverse flow where the recirculation intensity decreases as the stenosis ratio increases, and thus the WSS decreases too. Not only the WSS represents a valid variable to study atherosclerosis progression, it is also important to analyze the variation of this parameter along the cardiac cycle or its spatial variation. Figure 9 shows the maximum TAWSS varying for the four different geometrical parameters considered in this work. The only parameter that really influences TAWSS is the stenosis ratio (Fig. 9.b), where a clear negative linear correlation appears between TAWSS and sr $(r=-0.988, p=0.002)$, varying from $22.3 \mathrm{~Pa}$ to $9.28 \mathrm{~Pa}$ for $s r=46.7 \%$ and $s r=66.7 \%$, respectively. The TAWSS increases with a decreasing $s r$ parameter. The TAWSS presents a non-symmetric distribution respect the central plane, reaching higher values in the post-stenotic zone. The other three parameters, cap thickness, lipic core length or lipic core width, do not show any significant correlation with TAWSS, with slight variations of 5$10 \%$ due to small changes in the location of the maximum TAWSS point. Other hemodynamical parameters usually referred in the literature as related with plaque apparition or progressions, OSI, RRT and AWSSG were also studied. The dependence of these parameters with the morphological variables was analyzed. Almost any correlation was found among any of these hemodynamical parameters and any geometrical factor. The better correlation appeared between the stenosis ratio and the hemodynamical parameters and it is depicted in Figure 10, where the maximum value of the OSI, RRT and AWSSG is represented. The correlation coefficient for 
RRT and AWSSG was $r=-0.457, r=-0.584$. The other three morphological parameters do not significantly affect the hemodynamics of the vessel.

\section{Discussion}

Plaque stress has been studied for several decades as a 2D structure. Fully coupled 3D plaque stress in both structure dynamics and hemodynamics are only recently explored, demonstrating better performance than 2D models [41]. The extremely high stress locations in the fibrous cap and shoulders regions have been mostly considered to be main factors responsible for plaque rupture. Collapse of severe but compliant stenoses due to negative transmural pressures may produce highly concentrated compressive stresses from buckling of the wall with bending deformation, preferentially involving plaque edges, and theoretically, this could contribute to plaque disruption [44]. Furthermore, the propagating pulse wave causes cyclic changes in lumen size and shape with deformation and bending of plaques, particularly the soft ones, and therefore, the repetitive deformations caused by the cardiac cycle may play an important role in lesion stability [45]. Other features such as great arterial deformations, high wall shear stress values at the arterial wall or blood flow vorticity could also contribute to the atheroma plaque instability [44,45]. Moreover, some variables, which can be obtained by FSI analysis, such as the low WSS and/or time dependent vorticity has been linked to the initiation of atherosclerotic lesions $[12,13]$. Consequently, the importance of cardiovascular FSI modeling can be demonstrated since this kind of analysis allows an accurate prediction of not only potential sites of vulnerable plaque rupture through the stress distribution of the atherosclerotic artery model but also the initiation and progression of the lesions related to the WSS pattern. Additionally, the effect of complex arterial loading experienced under pulsatile flow conditions has been shown neglectable for the accurately prediction of the MPS, as we have found similar MPS contour maps obtained by the FSI and pure structural analysis, respectively. This means that regarding the MPS distribution along the vessel model, the FSI analysis leads to similar conclusions than those obtained for the pure structural models [6]. The fibrous cap thickness is 
the most influential parameter in terms of plaque vulnerability, increasing the MPS as this parameter decreases. The lipid core length and width are less influential parameters on plaque stability, however, they should be taken into account to assess atheroma plaque vulnerability risk. MPS increases as these parameters increase. Finally, the stenosis degree may not be a good predictor for plaque rupture risk, occurring a slight increase of MPS as this parameter increases. Furthermore, any of the 17 simulated models by FSI analysis has been predicted as vulnerable (MPS higher than the vulnerability threshold of $247 \mathrm{kPa}$ ) since the considered geometric models are built from combination of parameter values out of the vulnerability limits defined in Cilla et al. [6]. Moreover, as expected, the MPS distribution follows a tendency similar to the applied cardiac cycle. Maximal arterial deformation can be found in the non-stenotic zones of the model. However, the maximal principal strains should be observed at the critical zones (stenotic areas) in order to extract conclusions about atheroma plaque vulnerability. Then, the maximum deformation at these sites remains around $15 \%$ for all studied model. Furthermore, as for the maximum MPS, the maximum deformation increases as the stenosis ratio and lipid core width and length increase, and it decreases with the fibrous cap thickness. As mentioned in the introduction section, the WSS is believed to play an important role in the plaque initiation and development. Low WSS or highly oscillatory WSS on vessel segments appear to be at the highest risk for development of atherosclerosis, since these phenomena modulate the endothelial expression. This fact is due to complex mechanoreception and mechanotransduction processes, inducing an atherogenic endothelial phenotype and formation of an early atherosclerotic plaque [46]. Furthermore, some authors such as Groen et al. [47,43] proposed that the high WSS in the fibrous cap could produce the rupture of vulnerable plaques. Additionally, because WSS is affected by luminal dimensions, some authors have argued that the local WSS can be estimated from purely anatomic measures such as angiography or intravascular ultrasound [12]. In this study, high WSS values have been found in the central part of the stenosis, which could exacerbate the atheroma plaque unstability. On the contrary, low WSS values are identified in the post stenotic region, 
zones of flow recirculation, providing an indication of possible areas of atheroma plaque initiation. As expected, the distribution of this variable varies just with the stenosis degree. Therefore, the stenosis ratio which was defined as not influential in term of plaque stability, plays an important role in the appearance and progression of atheroma plaque. TAWSS is mainly affected by stenosis ratio, while the other hemodynamics factors, OSI, RRT and AWSSG do not show a clear correlation with and morphological parameter. As stated in literature non-stenotic vulnerable plaques are associated with low shear stress values, which can promote inflammation and influence plaque stability. This contrasts with stenotic high-risk plaques that are typically exposed to high shear stress. For this reason, co-localization of high stresses at the lumen wall and low plaque strength may be considered as a novel future marker for identification of vulnerable plaques [48].

\section{Conclusion}

A FSI model of an idealized straight coronary artery was created and analyzed under several parametric geometrical variations in order to find which parameters are more relevant in terms of plaque initiation, progression and rupture. Several hemodynamic and mechanical indexes were computed. The simulations results show that hemodynamics and mechanical factors are not directly coupled and mutually influenced. Due to the increased stiffness of the artery at the stenosis, the hemodynamics of the artery resulted not affected by the compliance. The fluid dynamics parameters seemed to be dependent only on the stenosis ratio. On other hand, we found that the mechanical variables such as maximum principal stress and strain were dependent on the geometry of the artery. Furthermore, a comparison between FE and FSI results shows no influence of these variable on the arterial hemodynamics. Therefore, the results of the present study suggest the possibility to perform FE and CFD separately in the future, rather using FSI for idealized models of a straight coronary artery. However, as discussed, co-localization of high tensile wall stresses and low plaque strength associated to endothelial shear stress may be important in the future for identifying vulnerable plaques. 


\section{Conflict of interest}

None of the authors of this work has conflict of interest with other people and organizations.

\section{Acknowledgments}

The authors gratefully acknowledge the support of the Spanish Ministry of Science and Technology through the research project DPI2013-44391-P. The support of the Instituto de Salud Carlos III (ISCIII) through the CIBER-BBN initiative is highly appreciated. Finally, we also thank the Diputación General de Aragón (DGA) for the financial support to Dr. M. Cilla through the grant B137/09.

\section{References}

[1] A. J. Lusis, Atherosclerosis, Nature 407 (6801) (2000) 233-241.

[2] A. C. Van der Wal, A. E. Becker, Atherosclerotic plaque rupture - Pathologic basis of plaque stability and instability, Cardiovascular Research 41 (1999) 334344 .

[3] G. Finet, J. Ohayon, G. Rioufol, Biomechanical interaction between cap thickness, lipid core composition and blood pressure in vulnerable coronary plaque: Impact on stability or instability, Coronary Artery Disease 15 (2004) $13-20$.

[4] R. Krishna Kumar, K. R. Balakrishnan, Influence of lumen shape and vessel geometry on plaque stresses: Possible role in the increased vulnerability of a remodelled vessel and the shoulder of a plaque, Heart 91 (2005) 1459-1465.

[5] J. Ohayon, G. Finet, A. M. Gharib, D. A. Herzka, P. Tracqui, J. Heroux, G. Rioufol, M. S. Kotys, A. Elagha, R. I. Pettigrew, Necrotic core thickness and positive arterial remodeling index: Emergent biomechanical factors for evaluating the risk of plaque rupture, American Journal of Physiology - Heart and Circulatory Physiology 295 (2008) 717-727. 
[6] M. Cilla, E. Peña, M. A. Martínez, 3D computational parametric analysis of eccentric atheroma plaque. Influence of axial residual stresses, Biomechanics and Modeling in Mechanobiology 11 (7) (2012a) 1001-1013.

[7] R. Virmani, F. D. Kolodgie, A. P. Burke, A. Farb, S. M. Schwartz, Lessons from sudden coronary death: A comprehensive morphological classification scheme for atherosclerotic lesions, Arteriosclerosis, Thrombosis, and Vascular Biology 20 (2000) 1262-1275.

[8] D. Bluestein, Y. Alemu, I. Avrahami, M. Gharib, K. Dumont, J. J. Ricotta, S. Einav, Influence of microcalcifications on vulnerable plaque mechanics using FSI modelling, Journal of Biomechanics 41 (2008) 1111-1118.

[9] J. F. Wenk, P. Papadopoulos, T. I. Zohdi, Numerical modeling of stress in stenotic arteries with microcalcifications: A micromechanical approximation, ASME Journal of Biomechanical Engineering 132 (9) (2010) 091011.

[10] J. F. Wenk, Numerical modeling of stress in stenotic arteries with microcalcifications: A parameter sensitivity study, ASME Journal of Biomechanical Engineering 133 (2011) 014503.

[11] M. Cilla, D. Monterde, E. Peña, M. A. Martínez, Does microcalcification increase the risk of rupture?, Proceedings of the Institution of Mechanical Engineers, Part H, Journal of Engineering in Medicine 227 (5) (2013) 588-599.

[12] D. A. Steinman, Image-based computational fluid dynamics: A new paradigm for monitoring hemodynamics and atherosclerosis, Current Drug Targets Cardiovascular \& Hematological Disorders 4 (2) (2004) 183-197.

[13] S. Chien, Effects of disturbed flow on endothelial cells, Annals of Biomedical Engineering 36 (4) (2008) 554-562.

[14] C. G. Caro, J. M. Fitz-Gerald, R. C. Schroter, Atheroma and arterial wall shear. Observation and proposal of a shear dependent mass transfer mechanism for atherogenesis, Proceedings of the Royal Society B: Biological Sciences 177 (1046) (1971) 109-159. 
[15] R. Torii, N. Wood, N. Hadjiloizou, A. Dowsey, A. Wright, A. Hughes, J. Davies, D. Francis, J. Mayet, G. Yang, S. McG, X. X. Thom, Fluid-Structure Interaction analysis of a patient-specific right coronary artery with physiological velocity and pressure waveforms, Communications in Numerical Methods in Engineering 55 (2009) 565-580.

[16] M. Malvè, A. García, J. Ohayon, M. A. Martínez, Unsteady blood flow and mass transfer of a human left coronary artery bifurcation: FSI vs. CFD, International Communications in Heat and Mass Transfer 39 (6) (2012) 745-751.

[17] S. Hyun, C. Kleinstreuer, J. P. Archie, Hemodynamics analyses of arterial expansions with implications to thrombosis and restenosis, Medical Engineering \& Physics 22 (1) (2000) 13-27.

[18] T. Asakura, T. Karino, Flow patterns and spatial distribution of atherosclerotic lesions in human coronary arteries, Circulation Research 66 (4) (1990) 10451066.

[19] J. Soulis, M. Fermakis, G. Giannoglou, G. Louridas, Wall shear stress in normal left coronary artery tree, Journal of Biomechanics 39 (2006) 742-749.

[20] G. Giannoglou, J. Soulis, T. Fermakis, G. Zioupos, G. Giannakoulas, G. Louridas, Wall shear stress differences between proximal and distal left coronary artery bifurcations, Abstract of European Heart Journal 1 (2002) 686.

[21] L. Goubergrits, E. Wellnhofer, U. Kertzscher, K. Affeld, C. Petz, C. Hege, Coronary artery wss profiling using a geometry reconstruction based on biplane angiography, Annals of Biomedical Engineering 37 (4) (2007) 682-691.

[22] Z. Y. Li, S. Howarth, R. A. Trivedi, J. M. U-King-Im, M. J. Graves, A. Brown, L. Wang, J. H. Gillard, Stress analysis of carotid plaque rupture based on in vivo high resolution MRI, Journal of Biomechanics 39 (14) (2006b) 2611-2622.

[23] S. A. Kock, J. V. Nygaard, N. Eldrup, E.-T. Fründ, A. Klaerke, W. P. Paaske, E. Falk, W. Y. Kim, Mechanical stresses in carotid plaques using MRI-based Fluid-Structure Interaction models, Journal of Biomechanics 41 (2008) 16511658. 
[24] S. Z. Zhao, B. Ariff, Q. Long, A. D. Hughes, S. A. Thom, A. V. Stanton, $\mathrm{X}$. Y. Xu, Inter-individual variations in wall shear stress and mechanical stress distributions at the carotid artery bifurcation of healthy humans, Journal of Biomechanics 35 (10) (2002) 1367-1377.

[25] R. Fan, D. L. Tang, C. Yang, J. Zheng, R. Bach, L. Wang, D. Muccigrosso, K. Billiar, J. Zhu, G. S. Ma, A. Maehara, G. S. Mintz, Human coronary plaque wall thickness correlated positively with flow shear stress and negatively with plaque wall stress: an IVUS-based fluid-structure interaction multi-patient study, Biomedical Engineering Online 13 (2014) 1-14.

[26] X. Y. Huang, C. Yang, J. Zheng, R. Bach, D. Muccigrosso, P. K. Woodard, D. L. Tang, Higher critical plaque wall stress in patients who died of coronary artery disease compared with those who died of other causes: A 3D FSI study based on ex vivo MRI of coronary plaques, Journal of Biomechanics 47 (2014) $432-437$.

[27] M. H. Kural, M. Cai, D. Tang, T. Gwyther, J. Zheng, K. L. Billiar, Planar biaxial characterization of diseased human coronary and carotid arteries for computational modeling, Journal of Biomechanics 45 (2012) 790-798.

[28] D. Tang, C. Yang, J. Zheng, P. K. Woodard, J. E. Saffitz, G. A. Sicard, T. K. Pilgram, C. Yuan, Quantifying effects of plaque structure and material properties on stress distributions in human atherosclerotic plaques using 3D FSI models, ASME Journal of Biomechanical Engineering 127 (2005) 1185-1194.

[29] D. Tang, C. Yang, J. Zheng, P. Woodard, J. Saffitz, J. Petruccelli, G. Sicard, C. Yuan, Local maximal stress hypothesis and computational plaque vulnerability index for atherosclerotic plaque assessment, Annals of Biomedical Engineering 33 (2005) 1789-1801.

[30] D. Tang, C. Yang, S. Mondal, F. Liu, G. Canton, T. S. Hatsukami, C. Yuan, A negative correlation between human carotid atherosclerotic plaque progression and plaque wall stress: In Vivo MRI-based 2D/3D FSI models, Journal of Biomechanics 41 (4) (2008b) 727-736. 
[31] D. Tang, Z. Teng, G. Canton, C. Yang, M. Ferguson, X. Huang, J. Zheng, P. K. Woodard, C. Yuan, Sites of rupture in human atherosclerotic carotid plaques are associated with high structural stresses: An in vivo MRI-based 3D FluidStructure Interaction study, Stroke 40 (2009) 3258-3263.

[32] M. X. Li, J. J. Beech-Brandt, L. R. John, P. R. Hoskins, W. J. Easson, Numerical analysis of pulsatile blood flow and vessel wall mechanics in different degrees of stenosis, Journal of Biomechanics 40 (16) (2007) 3715-3724.

[33] J. Ohayon, A. M. Gharib, A. García, J. Heroux, S. K. Yazdani, M. Malvè, P. Tracqui, M. A. Martínez, M. Doblaré, G. Finet, R. I. Pettigrew, Is arterial wall-strain stiffening an additional process responsible for atherosclerosis in coronary bifurcations?: An in vivo study based on dynamic ct and mri, American Journal of Physiology - Heart and Circulatory Physiology 301 (2011) 1097-1106.

[34] A. Versluis, A. J. Bank, W. H. Douglas, Fatigue and plaque rupture in myocardial infarction, Journal of Biomechanics 39 (2006) 339-347.

[35] G. A. Holzapfel, C. T. Gasser, G. Sommer, P. Regitnig, Determination of the layer-specific mechanical properties of human coronary arteries with nonatherosclerotic intimal thickening, and related constitutive modelling, American Journal of Physiology - Heart and Circulatory Physiology 289 (2005b) 20482058.

[36] B. M. Johnston, P. R. Johnston, S. Corney, D. Kilpatrick, Non-newtonian blood flow in human right coronary arteries: Transient simulations, Journal of Biomechanics 39 (6) (2006) 1116-1128.

[37] A. Valencia, M. Villanueva, Unsteady flow and mass transfer in models of stenotic arteries considering Fluid-Structure Interaction, International Communications in Heat and Mass Transfer 33 (2006) 966-975.

[38] A. Valencia, F. Baeza, Numerical simulation of Fluid-Structure Interaction in stenotic arteries considering two layer nonlinear anisotropic structural model, International Communications in Heat and Mass Transfer 36 (2009) 137-142. 
[39] J. E. Davies, Z. I. Whinnett, D. P. Francis, C. H. Manisty, J. Aguado-Sierra, K. Willson, R. A. Foale, I. S. Malik, A. D. Hughes, K. H. Parker, J. Mayet, Evidence of a dominant backward-propagating suction wave responsible for diastolic coronary filling in humans, attenuated in left ventricular hypertrophy, Circulation 113 (14) (2006) 1768-1778.

[40] H. M. Loree, R. D. Kamm, R. G. Stringfellow, R. T. Lee, Effects of fibrous cap thickness on peak circumferential stress in model atherosclerotic vessels, Circulation Research 71 (1992) 850-858.

[41] J. Ohayon, G. Finet, F. Treyve, G. Rioufol, O. Dubreuil., A three dimensional finite element analysis of stress distribution in a coronary atherosclerotic plaque: In-vivo prediction of plaque rupture location, Biomechanics Applied to Computer Assisted Surgery 17 (2005) 225-241.

[42] X. He, D. N. Ku, Pulsatile flow in the human left coronary artery bifurcation: average conditions, Journal of Biomechanical Engineering 118 (1996) 74-82.

[43] H. C. Groen, F. J. Gijsen, A. Van Der Lugt, M. S. Ferguson, T. S. hatsukami, A. F. Van Der Steen, C. Yuan, J. J. Wentzel, High shear stress influences plaque vulnerability, Netherlands Heart Journal 16 (7) (2008) 280-283.

[44] T. Aoki, D. N. Ku, Collapse of diseased arteries with eccentric cross section, Journal of Biomechanics 26 (1993) 133-142.

[45] K. Mizushige, M. Reisman, M. Buchbinder, H. Dittrich, A. N. DeMaria, Atheroma deformation during the cardiac cycle: Evaluation by intracoronary ultrasound, Circulation 88 - Supplement 1 (1993) 550.

[46] Y. S. Chatzizisis, A. U. Coskun, M. Jonas, E. R. Edelman, C. L. Feldman, P. H. Stone, Role of endothelial shear stress in the natural history of coronary atherosclerosis and vascular remodeling, Journal of the American College of Cardiology 49 (25) (2007) 2379-2393.

[47] H. C. Groen, F. J. Gijsen, A. Van Der Lugt, M. S. Ferguson, T. S. hatsukami, A. F. Van Der Steen, C. Yuan, J. J. Wentzel, Plaque rupture in the carotid 
artery is localized at the high shear stress region: A case report, Stroke 38 (8) (2007) 2379-2381.

[48] B. R. Kwak, M. Ba, M. L. Bochaton-Piallat, G. Caligiuri, M. J. A. P. Daemen, P. F. Davies, I. E. Hoefer, P. Holvoet, H. Jo, R. Krams, S. Lehoux, C. Monaco, S. Steffens, R. Virmani, C. Weber, J. J. Wentzel, P. C. Evans, Biomechanical factors in atherosclerosis: mechanisms and clinical implications, European Heart Journal (2014) 1-12.
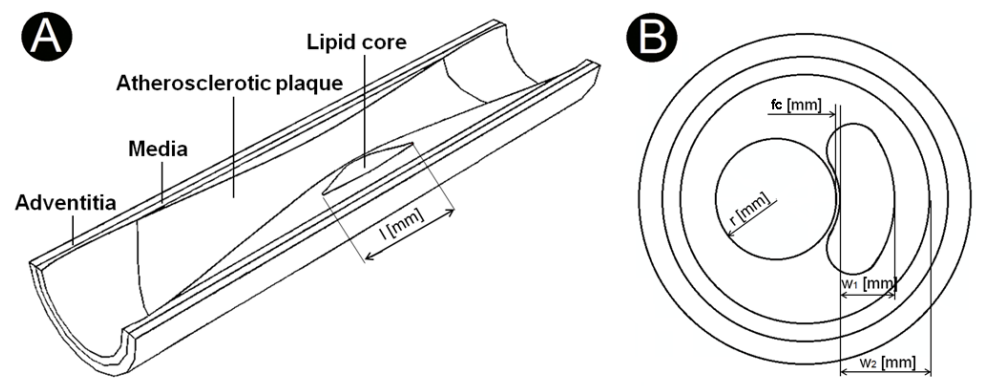

Fig. 1. Idealized geometry of theatherosclerotic artery (Transversal (A) and central cross section (B).

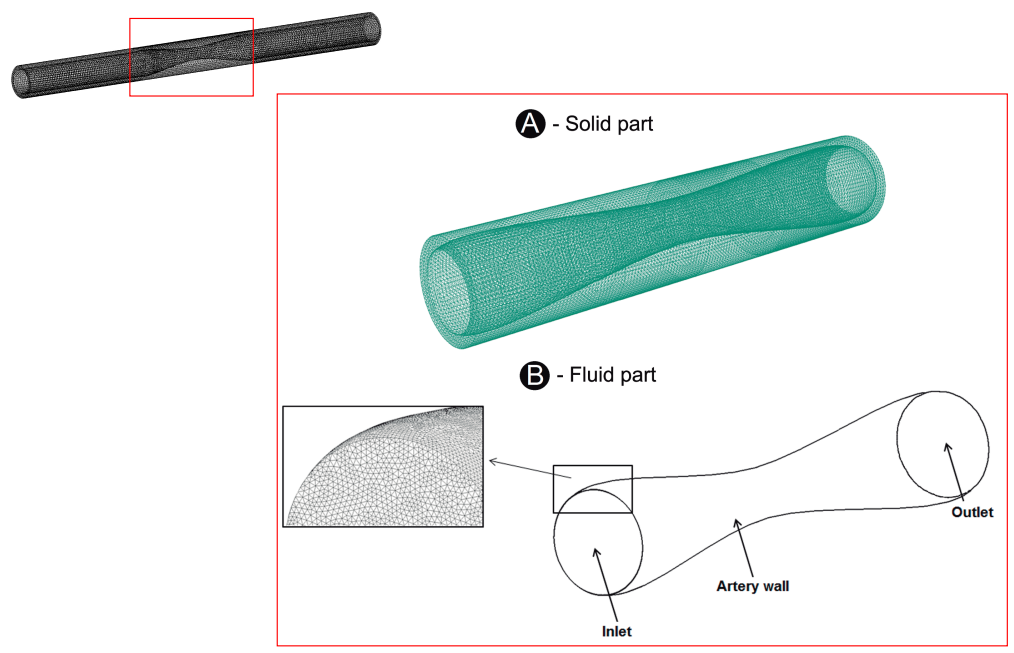

Fig. 2. Computational mesh. 

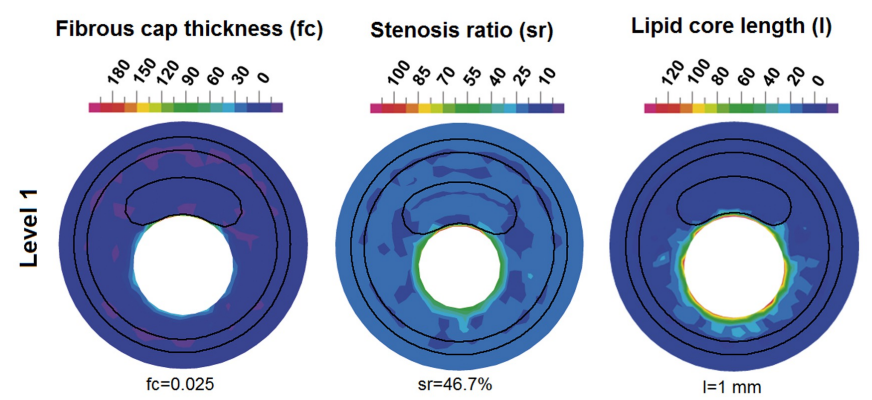

Lipid core width (w)
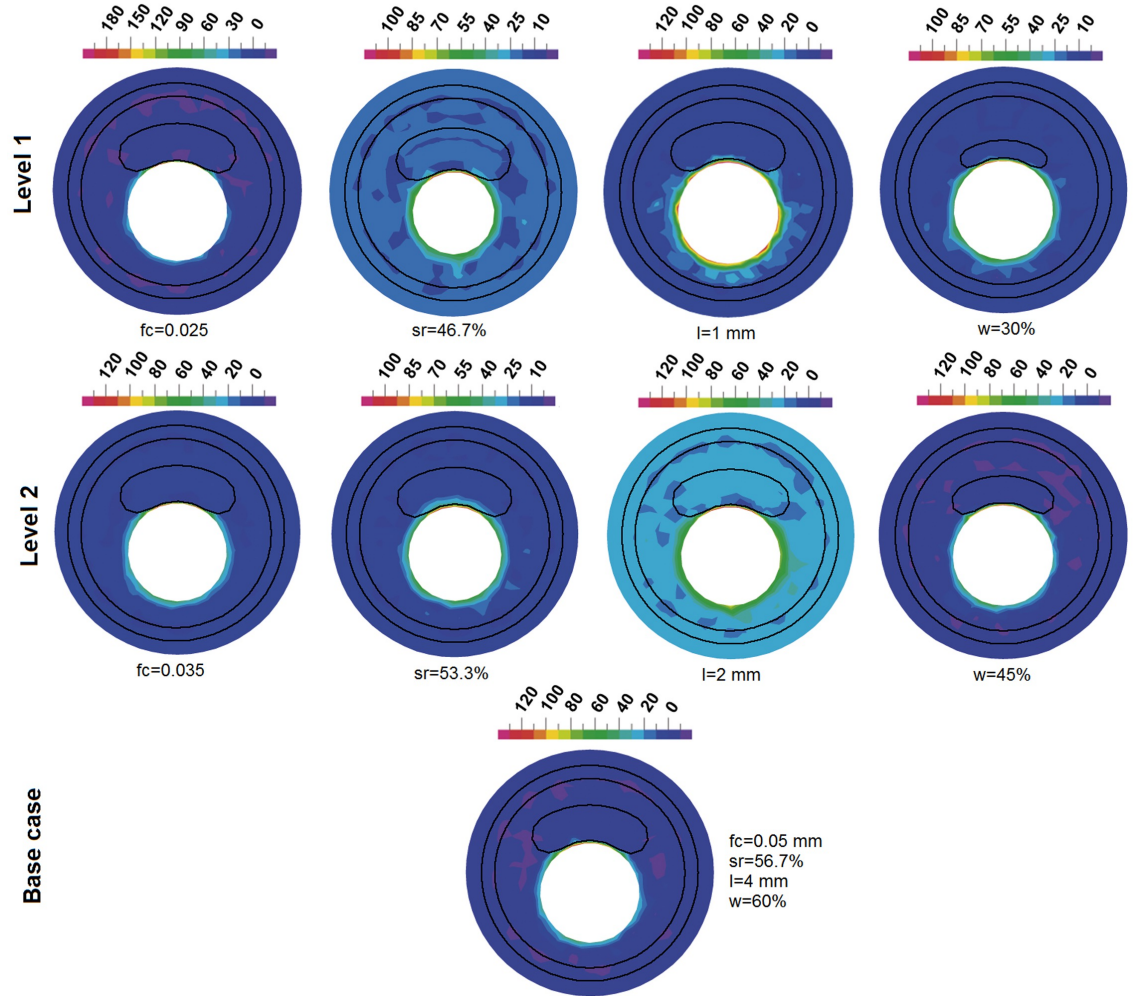

$\mathrm{w}=30 \%$

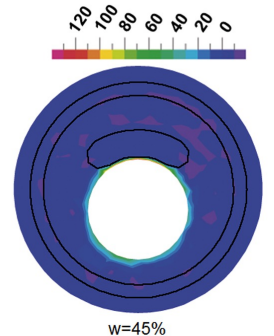

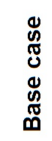
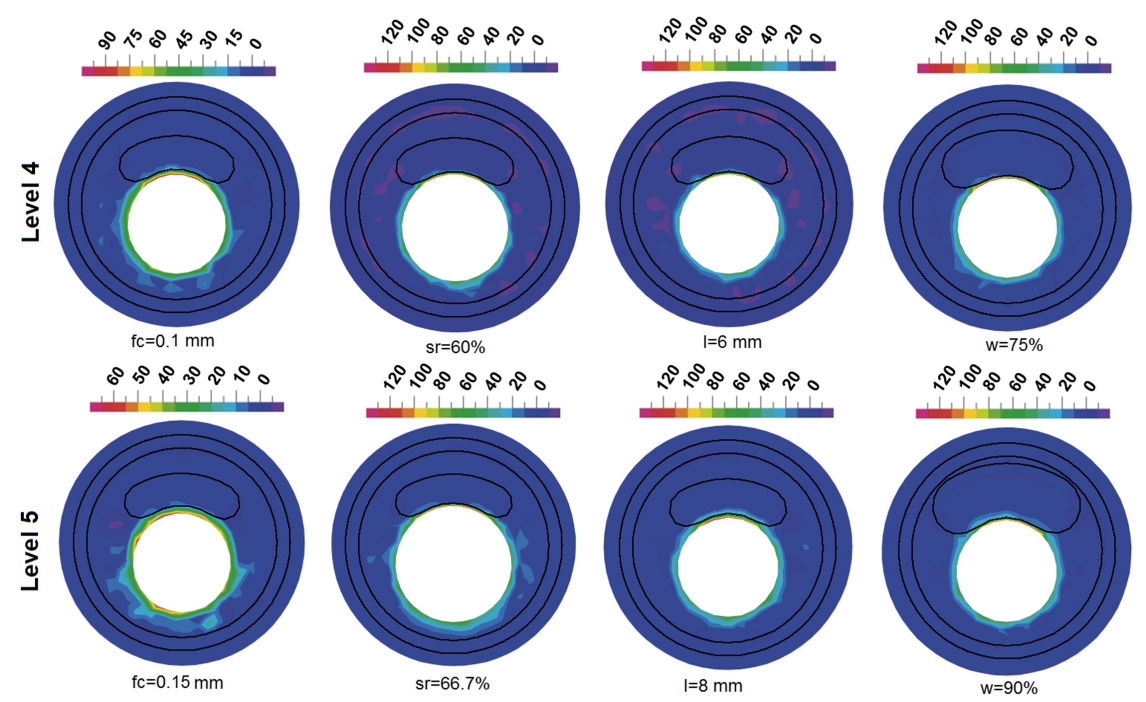

Fig. 3. Contour maps of the MPS at the central cross section of each of the 17 models studied. 

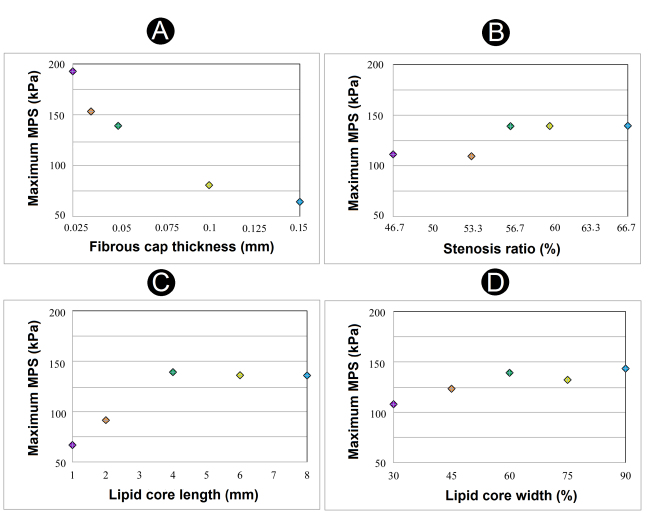

Fig. 4. Maximum MPS for each of the 17 models at peak pressure $(t=0.36 \mathrm{~s})$.

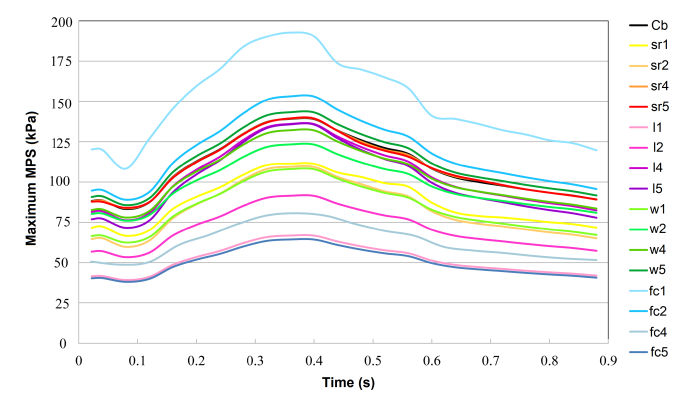

Fig. 5. MPS variation along a physiological coronary flow waveform in the central section of each of the 17 models. 


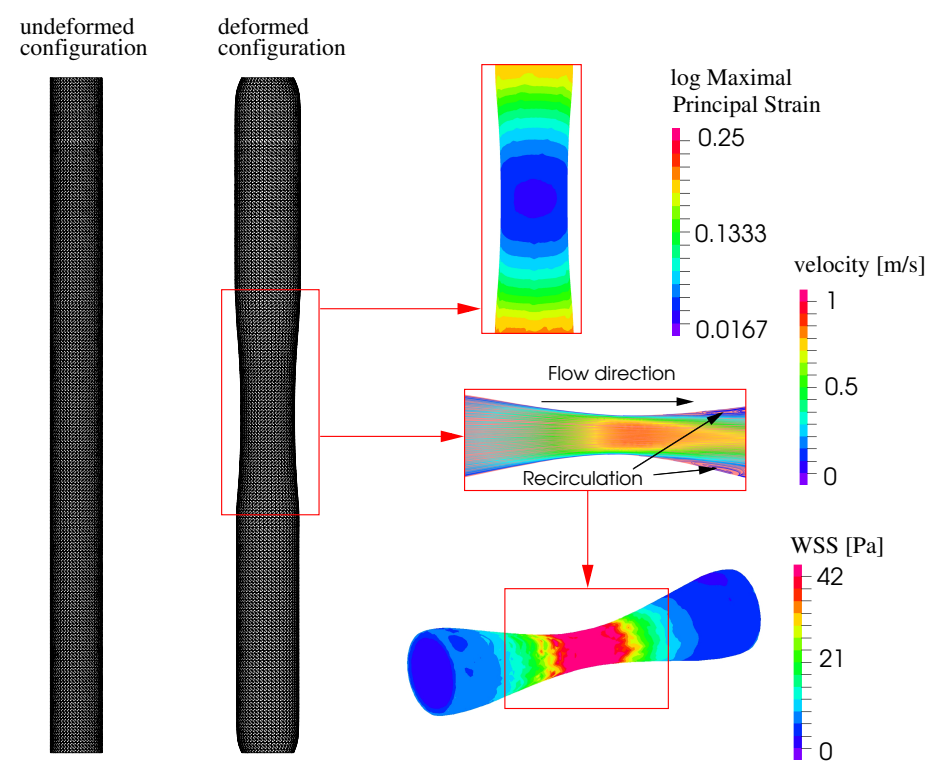

Fig. 6. Deformed and undeformed configuration belonging to the reference case and its corresponding contour maps of the maximal principal strain in logarithmic scale at peak pressure $(t=0.36 \mathrm{~s})$, wall shear stress and velocity field at peak flow $(t=0.56 s)$.

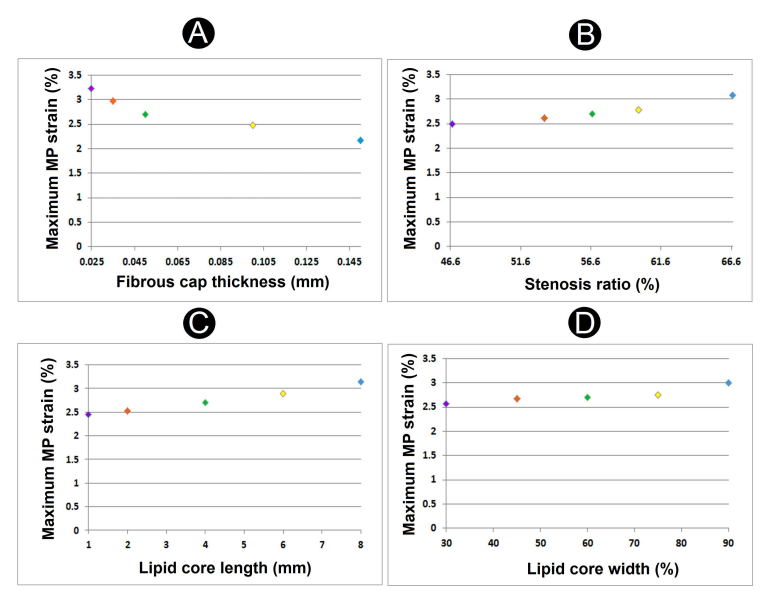

Fig. 7. Maximum Maximal Principal Strain (measured in percentage) of each studied model in the internal surface of the atheroma plaque. 


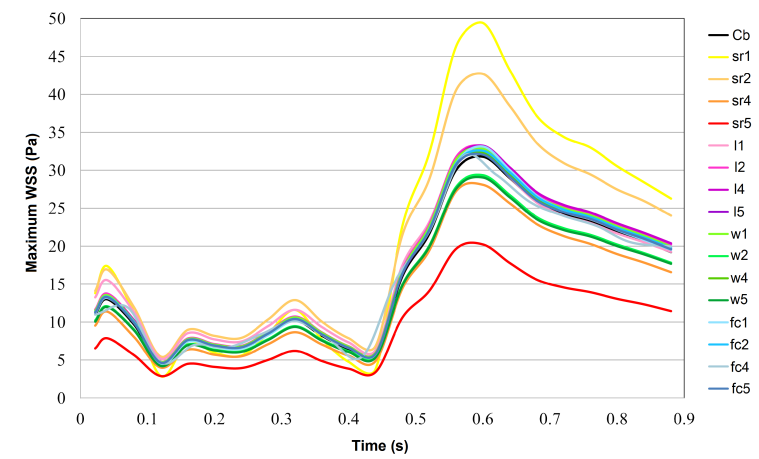

Fig. 8. Maximum WSS along a cardiac cycle for the 17 simulated models.

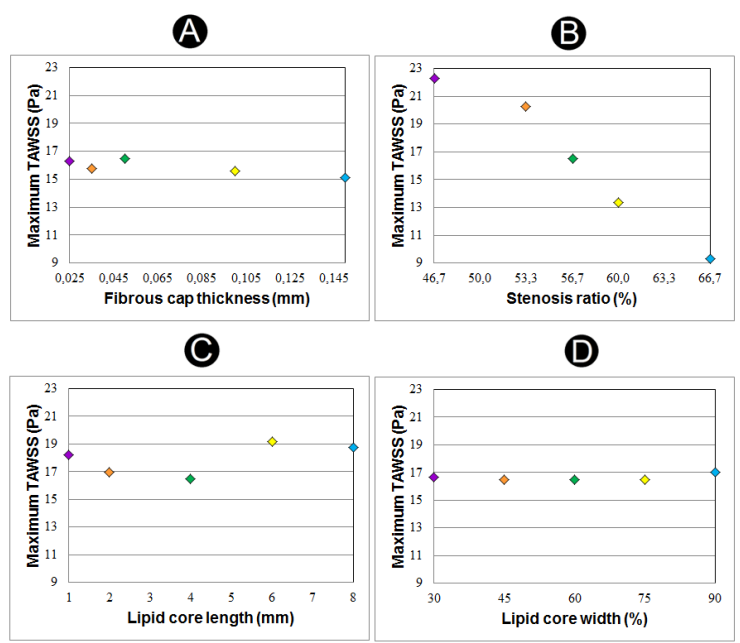

Fig. 9. Maximum TAWSS for the 17 simulated models.

A

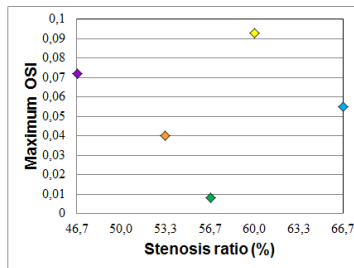

C
B

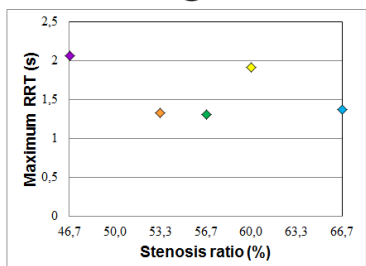

0

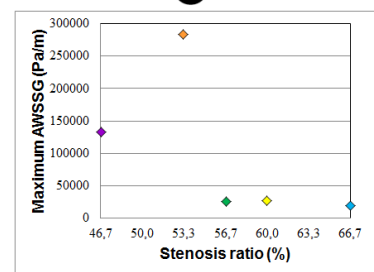

Fig. 10. Maximum OSI, RRT and AWSSG for the different stenosis ratio models. 